\title{
A New Heuristic for the Convex Quadratic Programming Problem
}

\author{
Elias Munapo1, Santosh Kumar ${ }^{2 *}$ \\ ${ }^{1}$ Graduate School of Business and Leadership, University of KwaZulu-Natal, Westville Campus, Durban, \\ South Africa \\ ${ }^{2}$ Department of Mathematics and Statistics, University of Melbourne, Parkville, Australia \\ Email: emunapo@gmail.com, ${ }^{*}$ skumar@ms.unimelb.edu.au
}

Received 9 July 2015; accepted 30 August 2015; published 2 September 2015

Copyright @ 2015 by authors and Scientific Research Publishing Inc.

This work is licensed under the Creative Commons Attribution International License (CC BY).

http://creativecommons.org/licenses/by/4.0/

(c) (i) Open Access

\begin{abstract}
This paper presents a new heuristic to linearise the convex quadratic programming problem. The usual Karush-Kuhn-Tucker conditions are used but in this case a linear objective function is also formulated from the set of linear equations and complementarity slackness conditions. An unboundedness challenge arises in the proposed formulation and this challenge is alleviated by construction of an additional constraint. The formulated linear programming problem can be solved efficiently by the available simplex or interior point algorithms. There is no restricted base entry in this new formulation. Some computational experiments were carried out and results are provided.
\end{abstract}

\section{Keywords}

Convex Quadratic Programming, Linear Programming, Karush-Kuhn-Tucker Conditions, Simplex Method, Interior Point Method

\section{Introduction}

There are so many real life applications for the convex quadratic programming (QP) problem. The applications include portfolio analysis, structural analysis, discrete-time stabilisation, optimal control, economic dispatch and finite impulse design; see [1]-[3]. Some of the methods for solving the convex quadratic problem are active set, interior point, branch and bound, gradient projection, and Lagrangian methods, see [4]-[9] for more information on these methods.

In this paper we present a new heuristic to linearise the convex quadratic programming problem. The usual Karush-Kuhn-Tucker conditions are still used but in this case a linear objective function is also formulated from

\footnotetext{
${ }^{*}$ Corresponding author.
} 
the set of linear objective function equations and the complementary slackness conditions. There is an unboundedness challenge that is associated with the proposed linear formulation. To alleviate this challenge, an additional constraint is constructed and added to the linear formulation. The new linear formulation can be solved efficiently by the available simplex and interior point algorithms. There is no restricted base entry in the proposed approach. The time consuming complementarity pivoting is no longer necessary. Some computational experiments have been carried out and the objective of the computational experiments was to determine CPU times of the:

1) Proposed heuristic;

2) Regularised Active Set Method Mae and Saunders [10];

3) Primal-Dual Interior Point Algorithm.

It may be noted that the proposed method is suitable only if the quadratic programming problem satisfies conditions (1) to (5) mentioned in Section 2.1.

\section{Mathematical Background}

\subsection{The Quadratic Programming Problem}

Let a quadratic programming (QP) problem be represented by (1).

Minimize $f(X)=C X^{\mathrm{T}}+\frac{1}{2} X Q X^{\mathrm{T}}$

Subject to:

$$
A X^{\mathrm{T}} \leq B^{\mathrm{T}}, X^{\mathrm{T}} \geq 0
$$

where

$$
\begin{gathered}
C=\left(c_{1}, c_{2}, \cdots, c_{n}\right), X=\left(x_{1}, x_{2}, \cdots, x_{n}\right), \boldsymbol{Q}=\left(\begin{array}{ccc}
q_{11} & \cdots & q_{1 n} \\
\vdots & \ddots & \vdots \\
q_{n 1} & \cdots & q_{n n}
\end{array}\right), \\
A=\left(\begin{array}{ccc}
a_{11} & \cdots & a_{1 n} \\
\vdots & \ddots & \vdots \\
a_{m 1} & \cdots & a_{m n}
\end{array}\right) \text { and } B=\left(b_{1}, b_{2}, \cdots, b_{m}\right) .
\end{gathered}
$$

It is assumed that:

1) Matrix $Q$ is a $n \times n$ symmetric and positive definite,

2) Function $f(X)$ is strictly convex,

3) The conditions $Y X^{\mathrm{T}}=0$ and $\lambda S^{\mathrm{T}}=0$ hold. Here $Y$ and $S$ are dual and primal slack variables, respectively.

4) Since constraints are linear then the solution space is convex, and

5) Any maximization quadratic problem can be changed into a minimization and vice versa.

When the function $f(X)$ is strictly convex for all points in the convex region then the quadratic problem has a unique local minimum which is also the global minimum [11].

\subsection{Karush-Kuhn-Tucker Conditions}

The convex quadratic programming problem has special features that we can capitalize on when solving. All constraints are linear and the only nonlinear expression is the objective function. Let the Lagrangian function for the QP problem be $L$ and in this case

$$
L=C X^{\mathrm{T}}+\frac{1}{2} X Q X^{\mathrm{T}}+\lambda\left(A X^{\mathrm{T}}-B^{\mathrm{T}}\right)
$$

where $\lambda=\left(\lambda_{1}, \lambda_{2}, \cdots, \lambda_{m}\right)$ and $\lambda^{\mathrm{T}} \geq 0$. In this case we exclude the non-negativity conditions $X^{\mathrm{T}} \geq 0$. If $Y=\left(y_{1}, y_{2}, \cdots, y_{n}\right), Y^{\mathrm{T}} \geq 0$ and $S=\left(s_{1}, s_{2}, \cdots, s_{m}\right), S \geq 0$ then the Karush-Kuhn-Tucker conditions as given in [11] for a local minimum are: 


$$
\begin{gathered}
Q X^{\mathrm{T}}+A^{\mathrm{T}} \lambda^{\mathrm{T}}-Y^{\mathrm{T}}=-C^{\mathrm{T}} \\
A X^{\mathrm{T}}+S^{\mathrm{T}}=B^{\mathrm{T}}, \\
Y^{\mathrm{T}}, S^{\mathrm{T}} \geq 0 .
\end{gathered}
$$

Complementary slackness conditions are given in (5) and are only satisfied at the optimal point. These conditions are:

$$
Y X^{\mathrm{T}}=0 \text { and } \lambda S^{\mathrm{T}}=0
$$

Note $Y$ and $S$ are $n$ and $m$ dimensional vectors representing the slack variables. At this stage, we are unable to apply the simplex algorithm due to restricted base entry and this makes the simplex method approximately 8 times slower than its full speed compared to its unrestricted basis version.

\subsection{Some Matrix Operations}

Suppose $D=\left(d_{1}, d_{2}, \cdots, d_{m}\right)$ and $E=\left(e_{1}, e_{2}, \cdots, e_{m}\right)$ are single row matrices of the same dimension $m$ and $H=\left(h_{i j}\right), i=1,2, \cdots, m$ and $j=(1,2, \cdots, m)$ is an $m \times m$ dimensional matrix, the following must hold.

$$
\begin{aligned}
D E^{\mathrm{T}} & =E D^{\mathrm{T}} \\
D H E^{\mathrm{T}} & =E H D^{\mathrm{T}}
\end{aligned}
$$

Equations (6) and (7) can be easily verified. These simple results are used to eliminate the complementary slackness conditions.

\section{Elimination of Complementary Slackness Conditions}

\subsection{Elimination of $Y X^{\mathrm{T}}=0$}

Pre-multiply (3) by $X$, we have:

$$
\begin{gathered}
X\left(Q X^{\mathrm{T}}+A^{\mathrm{T}} \lambda^{\mathrm{T}}-Y^{\mathrm{T}}\right)=X\left(-C^{\mathrm{T}}\right) \\
X Q X^{\mathrm{T}}+X A^{\mathrm{T}} \lambda^{\mathrm{T}}-X Y^{\mathrm{T}}=-X C^{\mathrm{T}}
\end{gathered}
$$

From (6) $Y X^{\mathrm{T}}=X Y^{\mathrm{T}}$ and from (5) $X Y^{\mathrm{T}}=0$, then

$$
X Q X^{\mathrm{T}}+X A^{\mathrm{T}} \lambda^{\mathrm{T}}=-X C^{\mathrm{T}}
$$

By rearranging, we have

$$
X Q X^{\mathrm{T}}+X A^{\mathrm{T}} \lambda^{\mathrm{T}}+X C^{\mathrm{T}}=0
$$

\subsection{Elimination of $\lambda S^{T}=0$}

Pre-multiply (4) by $\lambda$, we have

$$
\begin{aligned}
& \lambda\left(A X^{\mathrm{T}}\right)+\lambda S^{\mathrm{T}}=\lambda B^{\mathrm{T}} \\
& \lambda\left(A X^{\mathrm{T}}\right)+\lambda S^{\mathrm{T}}=\lambda B^{\mathrm{T}}
\end{aligned}
$$

Since from (4) $\lambda S^{T}=0$, then

$$
\lambda A X^{\mathrm{T}}-\lambda B^{\mathrm{T}}=0
$$

\subsection{Elimination of $\lambda A X^{\mathrm{T}}$ or $X A^{\mathrm{T}} \lambda^{\mathrm{T}}$}

From (7), we have: $\lambda A X^{\mathrm{T}}=X A^{\mathrm{T}} \lambda^{\mathrm{T}}$, hence we can replace $X A^{\mathrm{T}} \lambda^{\mathrm{T}}$ by $\lambda A X^{\mathrm{T}}$ in relations (11) to get $\mathrm{t}(15)$ :

$$
X Q X^{\mathrm{T}}+\lambda A X^{\mathrm{T}}+X C^{\mathrm{T}}=0
$$


Subtracting (14) from (15), we obtain (16):

$$
X Q X^{\mathrm{T}}+X C^{\mathrm{T}}+\lambda B^{\mathrm{T}}=0
$$

\subsection{Linear Objective Function for the Quadratic Programming Problem}

Note that the expression in relation (13) is nonlinear but it can be rearranged so that the original quadratic programming objective function becomes a linear quantity. This can be achieved as follows:

Divide relation (16) by two, one obtains:

$$
\frac{1}{2} X Q X^{\mathrm{T}}+\frac{1}{2} X C^{\mathrm{T}}+\frac{1}{2} \lambda B=0
$$

Rearranging (17), we obtain (18):

$$
\frac{1}{2} X Q X^{\mathrm{T}}+X C^{\mathrm{T}}-\frac{1}{2} X C^{\mathrm{T}}+\frac{1}{2} \lambda B=0
$$

From (1) $f(X)=C X^{\mathrm{T}}+\frac{1}{2} X Q X^{\mathrm{T}}$, then, (18) becomes (19) or equivalently (20):

$$
\begin{gathered}
f(X)-\frac{1}{2} C X^{\mathrm{T}}+\frac{1}{2} \lambda B=0 \\
f(X)=\frac{1}{2} C X^{\mathrm{T}}-\frac{1}{2} \lambda B
\end{gathered}
$$

Thus the nonlinear objective function of the QP problem is now linearised but it creates a new challenge. We will discuss this in the next section.

\subsection{LP Equivalent to the Given QP}

From (1), (3) and (20), we have the following LP problem:

Minimize $\frac{1}{2} C X^{\mathrm{T}}-\frac{1}{2} \lambda B$

Subject to:

$$
Q X^{\mathrm{T}}+A^{\mathrm{T}} \lambda^{\mathrm{T}}-Y^{\mathrm{T}}=-C^{\mathrm{T}}, A X^{\mathrm{T}}+S^{\mathrm{T}}=B^{\mathrm{T}}, X^{\mathrm{T}}, \lambda^{\mathrm{T}}, Y^{\mathrm{T}}, S^{\mathrm{T}} \geq 0
$$

The minimisation problem (21) will have an unbounded solution due to negative coefficient of $\lambda$ in the objective function and negative coefficients of the slack variable $Y^{\mathrm{T}}$ in the constraints. These are the only source of unboundedness in the LP (21). Here, we let: $\omega=\frac{1}{2} \lambda B$ and $\phi=\mu Y^{\mathrm{T}}$ where $\mu=(1,1, \cdots, 1)$ a row vector of dimension $n$. The objective function is now modified as :

Minimize $\frac{1}{2} C X^{\mathrm{T}}-\frac{1}{2} \lambda B+l_{1} \omega+l_{2} \phi$, where $l_{1}$ and $l_{2}$ are very large constants relative to all other objective

coefficients. Both of these constants do not have to assume the same large values. A large number of experiments were done on a large number of quadratic programming problems and and it was observed that $l_{1} \ll l_{2}$ seems to work well. These experiments have been recorded later in this paper. In these experiments, it was noted that values of $l_{1}$ and $l_{2}$ on higher side can be as much as $l_{1}=1000\left(\left|c_{1}\right|+\left|c_{2}\right|+\cdots+\left|c_{n}\right|\right)$ and $l_{2}=50000\left(\left|c_{1}\right|+\left|c_{2}\right|+\cdots+\left|c_{n}\right|\right)$.

\subsection{Existence of a Linear Objective Function and Verification of Optimality}

The optimal solution of a convex quadratic programming model is unique and it satisfies the complementary conditions $Y X^{\mathrm{T}}=0$ and $\lambda S^{\mathrm{T}}=0$. The unique optimal solution to the convex quadratic programming is a corner point $P_{Q}$. Since the KKT conditions can be expressed as a linear objective function that can make $P_{Q}$ 
exist.

\section{Numerical Illustrations}

\subsection{Example 1}

Minimize $-8 x_{1}-16 x_{2}+x_{1}^{2}+4 x_{2}^{2}$

Subject to:

$$
x_{1}+x_{2} \leq 5, x_{1} \leq 3, x_{1}, x_{2} \geq 0
$$

This example was taken from Jensen and Bard (2012) without any modifications.

Linear formulation of the above QP

In this case we took $l_{1}=1000$ and $l_{2}=50000$ which are very large compared to coefficients 4; 8; 2.5; and

1.5. The LP problem is given by:

Maximize $4 x_{1}+8 x_{2}+2.5 \lambda_{1}+1.5 \lambda_{2}-1000 \omega-50000 \phi$

Subject to

$$
\begin{gathered}
2 x_{1}+\lambda_{1}+\lambda_{2}-y_{1}=8, \\
8 x_{2}+\lambda_{1}-y_{2}=16, x_{1}+x_{2}+s_{1}=5, \\
x_{1}+s_{2}=3, \\
2.5 \lambda_{1}+1.5 \lambda_{2}=\omega, \\
y_{1}+y_{2}=\phi \\
x_{1}, x_{2}, \lambda_{1}, \lambda_{2}, y_{1}, y_{2}, s_{1}, s_{2}, \omega, \phi \geq 0
\end{gathered}
$$

The solution of (23) by the simplex method is given by:

$$
x_{1}=3, x_{2}=2, \lambda_{2}=2, \omega=3, \lambda_{1}=y_{1}=y_{2}=s_{1}=s_{2}=\phi=0 .
$$

From the original QP objective function, we have the objective value given in (25).

$$
f(3,2)=-31
$$

Verification of optimality

The solution is optimal because complementary slackness conditions are satisfied as given in (26).

$$
\lambda_{1} s_{1}=\lambda_{2} s_{2}=y_{1} x_{1}=y_{2} x_{2}=0
$$

\subsection{Two More Examples}

Two more examples are solved to illustrate how the large constants are selected. Example 2 is taken from [12] and example 3 is from [13].

Example 2 from [12]

Minimize $\left(x_{1}-1\right)^{2}+\left(x_{2}-2.5\right)^{2}$

Subject to:

$$
-x_{1}+2 x_{2} \leq 2, x_{1}+2 x_{2} \leq 6, x_{1}-2 x_{2} \leq 2, x_{1}, x_{2} \geq 0
$$

The linear formulation of (27) becomes as given in (28).

Maximize $2 x_{1}+5 x_{2}+2 \lambda_{1}+6 \lambda_{2}+2 \lambda_{3}-1000 \omega-300000 \phi$

Such that:

$$
\begin{gathered}
2 x_{1}-\lambda_{1}+\lambda_{2}+\lambda_{3}-y_{1}=2, \\
2 x_{2}+2 \lambda_{1}+2 \lambda_{2}-2 \lambda_{3}-y_{2}=5, \\
-x_{1}+2 x_{2}+s_{1}=2, \\
x_{1}+2 x_{2}+s_{2}=6,
\end{gathered}
$$




$$
\begin{gathered}
x_{1}-2 x_{2}+s_{3}=2, \\
2 \lambda_{1}+5 \lambda_{2}=\omega, \\
y_{1}+y_{2}=\phi, \\
x_{1}, x_{2}, \lambda_{1}, \lambda_{2}, \lambda_{3}, y_{1}, y_{2}, s_{1}, s_{2}, s_{3}, \omega, \phi \geq 0
\end{gathered}
$$

The solution of (28) is as given in (29) and once again it is optimal as all complementary slackness conditions are satisfied.

$$
x_{1}=1.4, x_{2}=1.7, \lambda_{1}=0.8, s_{2}=1.2, s_{3}=4, \omega=1.8, \lambda_{2}=\lambda_{3}=y_{1}=y_{2}=s_{1}=\phi=0
$$

Example 3 from [13]

Minimize $-x_{1}-x_{2}+\frac{1}{2} x_{1}^{2}+x_{2}^{2}-x_{1} x_{2}$

Subject to: $-x_{1}+x_{2} \leq 3,-2 x_{1}-3 x_{2} \leq-6 ; x_{1}, x_{2} \geq 0$.

The linear formulation of the above example is given by (30).

Maximize $\frac{1}{2} x_{1}+\frac{1}{2} x_{2}+1.5 \lambda_{1}-3 \lambda_{2}-500 \omega-80000 \phi$

Subject to:

$$
\begin{gathered}
x_{1}-x_{2}+\lambda_{1}-2 \lambda_{2}-y_{1}=1 ;-x_{1}+2 x_{2}+\lambda_{1}-3 \lambda_{2}-y_{2}=1 ; \quad x_{1}+x_{2}+s_{1}=3 ; \\
2 x_{1}+3 x_{2}+s_{2}=6 ; 1.5 \lambda_{1}-3 \lambda_{2}=\omega ; y_{1}+y_{2}=\phi ; \text { where } x_{1}, x_{2}, \lambda_{1}, \lambda_{2}, y_{1}, y_{2}, s_{1}, s_{2}, \omega, \phi \geq 0 .
\end{gathered}
$$

The solution is given by: $x_{1}=1.8, x_{2}=1.2, \lambda_{1}=0.4, s_{2}=1.2, s_{3}=4, \omega=0.6, \lambda_{2}=y_{1}=y_{2}=s_{1}=\phi=0$. This solution is once again optimal as all complementary slackness conditions are satisfied.

\section{Computational Experiments}

A set of convex quadratic programming test problems are given in [14]. All these test problems were used in testing the proposed approach. The objective of the computational experiments was:

1) To determine that the LP optimal solution is also optimal to the given QP.

2) Compare CPU times of the proposed heuristics with Regularized Active Set Method and Primal-Dual Interior Point Method

The results are tabulated in Table 1. MATLAB R2013 (version 8.2) running on an Intel Pentium Dual desktop

Table 1. Computational experiments on the set of QP test problems.

\begin{tabular}{ccccccc}
\hline $\begin{array}{c}\text { Exp. } \\
\text { No. }\end{array}$ & Prob. & $\begin{array}{c}\text { No. of } \\
\text { Nanstraints } \\
(\mathrm{m})\end{array}$ & $\begin{array}{c}\text { No. of } \\
\text { Variables } \\
(\mathrm{n})\end{array}$ & $\begin{array}{c}\text { CPU secs } \\
\text { Proposed } \\
\text { Heurstic }\end{array}$ & $\begin{array}{c}\text { CPU secs } \\
\text { Active } \\
\text { Set }\end{array}$ & $\begin{array}{c}\text { CPU secs } \\
\text { Interior } \\
\text { Point }\end{array}$ \\
\hline 1 & AUG2D & 10,000 & 20,200 & 29.34 & 0.55 & 15.12 \\
2 & AUG2DC & 10,000 & 20,200 & 34.39 & 0.57 & 14.25 \\
3 & AUG2DCQP & 10,000 & 20,200 & 21.89 & 240.73 & 14.63 \\
4 & AUG2DQP & 10,000 & 20,200 & 37.19 & 228.72 & 14.76 \\
5 & AUG3D & 1000 & 3873 & 0.29 & 0.07 & 1.65 \\
6 & AUG3DC & 1000 & 3873 & 0.45 & 0.06 & 1.69 \\
7 & AUG3DCQP & 1000 & 3873 & 0.82 & 3.84 & 1.39 \\
8 & AUG3DQP & 1000 & 3873 & 0.53 & 5.02 & 1.56 \\
9 & BOYD1 & 18 & 93,261 & 89.67 & 214.24 & 107.10 \\
10 & BOYD2 & 0 & 93,263 & $*$ & 4168.93 & 2245.64 \\
\hline
\end{tabular}




\section{Continued}

\begin{tabular}{|c|c|c|c|c|c|c|}
\hline 11 & CONT-050 & 2401 & 2597 & 0.31 & 0.84 & 3.37 \\
\hline 12 & CONT-100 & 9801 & 10197 & 1.88 & 26.37 & 19.12 \\
\hline 13 & CONT-101 & 10,098 & 10197 & 112.7 & 35855.97 & 20.66 \\
\hline 14 & CONT-200 & 39,601 & 40,397 & 76.16 & 277.68 & 136.22 \\
\hline 15 & CONT-201 & 40,198 & 40,397 & 51.12 & 285.50 & 143.56 \\
\hline 16 & CONT-300 & 90,298 & 90,597 & 219.47 & 2449.75 & 721.76 \\
\hline 17 & CVXQP1-L & 5000 & 10,000 & 413.18 & 4516.19 & 2488.97 \\
\hline 18 & CVXQP1-M & 500 & 1000 & 0.65 & 5.94 & 1.85 \\
\hline 19 & CVXQP1-S & 50 & 100 & 0.01 & 0.04 & 0.21 \\
\hline 20 & CVXQP2-L & 2500 & 10,000 & 218.82 & 670.50 & 443.34 \\
\hline 21 & CVXQP2-M & 250 & 1000 & 0.63 & 4.24 & 1.52 \\
\hline 22 & CVXQP2-S & 25 & 100 & 0.02 & 0.04 & 0.26 \\
\hline 23 & CVXQP3-L & 7500 & 10,000 & 76.22 & 14069.08 & 736.74 \\
\hline 24 & CVXQP3-M & 750 & 1000 & 0.65 & 17.10 & 2.63 \\
\hline 25 & CVXQP3-S & 75 & 100 & 0.02 & 0.40 & 0.23 \\
\hline 26 & DPKLO1 & 77 & 133 & 0.02 & 0.01 & 0.17 \\
\hline 27 & DTOC3 & 9998 & 1499 & 74.1 & 0.32 & 107.10 \\
\hline 28 & DUAL1 & 1 & 85 & $\sim 0.00$ & 0.03 & 0.46 \\
\hline 29 & DUAL2 & 1 & 96 & $\sim 0.00$ & 0.01 & 0.43 \\
\hline 30 & DUAL3 & 1 & 111 & $\sim 0.00$ & 0.03 & 0.58 \\
\hline 31 & DUAL4 & 1 & 75 & $\sim 0.00$ & 0.01 & 0.37 \\
\hline 32 & DUALC1 & 215 & 9 & $\sim 0.00$ & 0.02 & 0.60 \\
\hline 33 & DUALC2 & 229 & 7 & $\sim 0.00$ & 0.01 & 0.44 \\
\hline 34 & DUALC5 & 278 & 8 & $\sim 0.00$ & 0.01 & 0.24 \\
\hline 35 & DUALC8 & 503 & 8 & $\sim 0.00$ & 0.02 & 0.70 \\
\hline 36 & EXDATA & 3001 & 3000 & 154.08 & $\sim 0.00$ & 200.08 \\
\hline 37 & GENH28 & 8 & 10 & $\sim 0.00$ & 7.76 & 0.05 \\
\hline 38 & GOULDQP2 & 349 & 699 & 0.31 & 0.87 & 0.78 \\
\hline 39 & GOULDQP3 & 349 & 699 & 0.08 & 0.01 & 0.65 \\
\hline 40 & HS118 & 17 & 15 & $\sim 0.00$ & $\sim 0.00$ & 0.14 \\
\hline 41 & HS21 & 1 & 2 & $\sim 0.00$ & $\sim 0.00$ & 0.14 \\
\hline 42 & HS268 & 5 & 5 & $\sim 0.00$ & 0.00 & 0.16 \\
\hline 43 & HS35 & 1 & 3 & $\sim 0.00$ & $\sim 0.00$ & 0.05 \\
\hline 44 & HS35MOD & 1 & 3 & $\sim 0.00$ & $\sim 0.00$ & 0.08 \\
\hline 45 & HS51 & 3 & 5 & $\sim 0.00$ & 0.00 & 0.05 \\
\hline 46 & HS52 & 3 & 5 & $\sim 0.00$ & $\sim 0.00$ & 0.04 \\
\hline
\end{tabular}




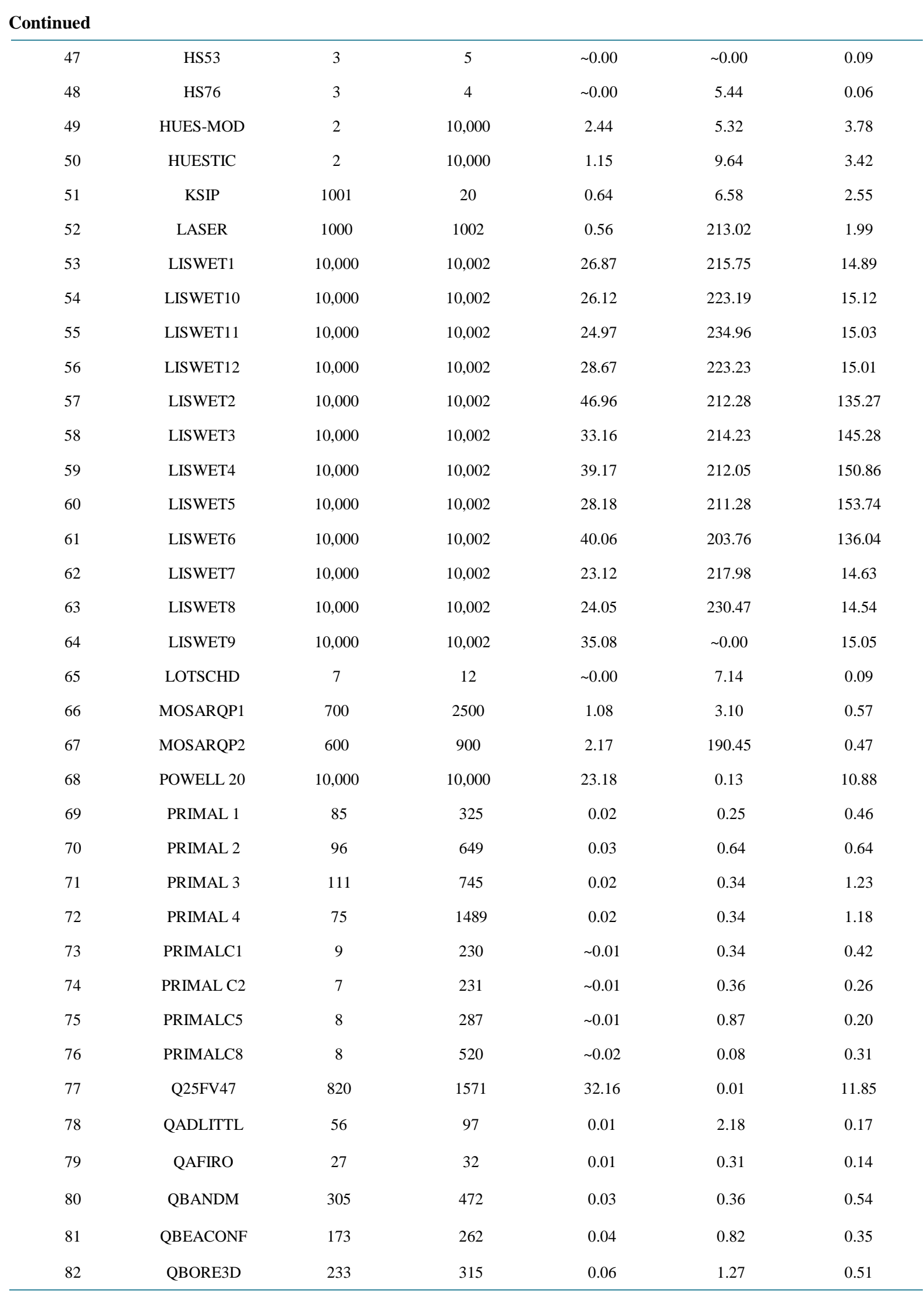




\section{Continued}

\begin{tabular}{|c|c|c|c|c|c|c|}
\hline 83 & QBRANDY & 220 & 249 & 0.05 & 1.86 & 0.35 \\
\hline 84 & QCAPRI & 271 & 353 & 0.06 & 4.22 & 1.18 \\
\hline 85 & QE226 & 223 & 282 & 0.04 & 5.39 & 0.50 \\
\hline 86 & QETAMACR & 400 & 688 & 0.06 & 1.26 & 1.86 \\
\hline 87 & QFFFFF80 & 524 & 854 & 0.07 & 6.24 & 1.54 \\
\hline 88 & QFORPLAN & 161 & 421 & 0.09 & 3.77 & 1.13 \\
\hline 89 & QGFRDXPN & 616 & 1092 & 1.06 & 8.42 & 2.04 \\
\hline 90 & QGROW15 & 300 & 645 & 0.08 & 0.84 & 1.32 \\
\hline 91 & QGROW22 & 440 & 946 & 0.05 & 0.66 & 2.09 \\
\hline 92 & QGROW7 & 140 & 301 & 0.04 & 0.10 & 0.81 \\
\hline 93 & QISRAEL & 174 & 142 & 0.02 & 3.68 & 0.71 \\
\hline 94 & QPCBLEND & 74 & 83 & 0.01 & 0.78 & 0.22 \\
\hline 95 & QPCBOE11 & 351 & 384 & 0.02 & 1.61 & 1.24 \\
\hline 96 & QPCBOE12 & 166 & 143 & 0.05 & 55.42 & 0.69 \\
\hline 97 & QPCSTAIR & 356 & 467 & 0.08 & $\sim 0.00$ & 0.86 \\
\hline 98 & QPILOTNO & 975 & 2172 & 8.15 & 0.02 & 4.76 \\
\hline 99 & QPTEST & 2 & 2 & $\sim 0.00$ & 0.30 & 0.08 \\
\hline 100 & QRECIPE & 91 & 180 & 0.08 & 2.58 & 0.41 \\
\hline 101 & QSC205 & 205 & 203 & 0.09 & 0.13 & 0.30 \\
\hline 102 & QSCAGR25 & 471 & 500 & 0.05 & 1.61 & 0.63 \\
\hline 103 & QSCAGR7 & 129 & 140 & 0.08 & 5.82 & 0.35 \\
\hline 104 & QSEFXM1 & 330 & 457 & 0.08 & 12.18 & 0.85 \\
\hline 105 & QSEFXM2 & 660 & 914 & 0.13 & 0.99 & 1.55 \\
\hline 106 & QSEFXM3 & 990 & 1371 & 1.12 & 5.25 & 2.38 \\
\hline 107 & QSCRPIO & 388 & 358 & 0.05 & 0.95 & 0.35 \\
\hline 108 & QSCRS8 & 490 & 1169 & 0.08 & 4.64 & 1.14 \\
\hline 109 & QSCSD1 & 77 & 760 & 0.87 & 29.78 & 6.87 \\
\hline 110 & QSCSd6 & 147 & 1350 & 0.09 & 2,71 & 0.68 \\
\hline 111 & QSCSD8 & 397 & 2750 & 0.04 & 22.41 & 1.13 \\
\hline 112 & QSETAP1 & 300 & 480 & 0.08 & 39.33 & 0.50 \\
\hline 113 & QSETAP2 & 1090 & 1880 & 0.23 & 1.58 & 1.17 \\
\hline 114 & QSETAP3 & 1480 & 2480 & 0.07 & 0.40 & 1.51 \\
\hline 115 & QSEBA & 515 & 1028 & 0.06 & 0.11 & 1.80 \\
\hline 116 & QSHARE1B & 117 & 225 & 0.04 & 23.37 & 0.44 \\
\hline 117 & QSHARE2B & 96 & 79 & 0.02 & 6.36 & 0.27 \\
\hline 118 & QSHELL & 536 & 1775 & 0.03 & 3.55 & 3.03 \\
\hline
\end{tabular}




\begin{tabular}{|c|c|c|c|c|c|c|}
\hline \multicolumn{7}{|l|}{ Continued } \\
\hline 119 & QSHIP04L & 402 & 2118 & 0.08 & 48.37 & 1.05 \\
\hline 120 & QSHIP04S & 402 & 1458 & 0.11 & 13.18 & 0.72 \\
\hline 121 & QSHIP08L & 778 & 4283 & 1.03 & 23.35 & 6.10 \\
\hline 122 & QSHIP08S & 778 & 2387 & 0.79 & 12.19 & 1.75 \\
\hline 123 & QSHIP12L & 1151 & 5247 & 1.26 & 1.90 & 11.76 \\
\hline 124 & QSHIP12S & 1151 & 2763 & 0.16 & 2.68 & 2.24 \\
\hline 125 & SIERRA & 1227 & 2036 & 0.12 & $\sim 0.00$ & 3.79 \\
\hline 126 & QSTAIR & 356 & 467 & 0.07 & 36.94 & 0.87 \\
\hline 127 & QSTANDAT & 359 & 1075 & 0.05 & 39.34 & 0.98 \\
\hline 128 & S268 & 5 & 5 & $\sim 0.00$ & 95.97 & 0.16 \\
\hline 129 & STADAT1 & 3999 & 2001 & 0.08 & 12.28 & 6.61 \\
\hline 130 & STADAT2 & 3999 & 2001 & 0.13 & 1.87 & 8.12 \\
\hline 130 & STADAT3 & 7999 & 4001 & 0.09 & $\sim 0.00$ & 14.16 \\
\hline 131 & STCQP1 & 2052 & 4097 & 0.15 & 759.14 & 1.87 \\
\hline 132 & STCQP2 & 2052 & 4097 & 0.06 & 0.61 & 3.89 \\
\hline 133 & TAME & 1 & 2 & $\sim 0.00$ & 9.38 & 0.03 \\
\hline 134 & UBH1 & 12,000 & 18,009 & 34.54 & $\sim 0.00$ & 62.83 \\
\hline 135 & VALUES & 1 & 202 & $\sim 0.00$ & 0.55 & 0.51 \\
\hline 136 & YAO & 2000 & 2002 & 0.08 & 0.57 & 3.66 \\
\hline 137 & ZECEVIC2 & 2 & 2 & $\sim 0.00$ & 240.73 & 0.83 \\
\hline
\end{tabular}

(Dual core G2020 2.9 GHz CPU, 2GB DDR3 1333 RAM) was used in these experiments. There were no advanced processing techniques embedded within the three methods. The set up time was excluded from the CPU times in all three methods. The zero $(\sim 0.00)$ means CPU time is less than 0.01 second. In all the test problems, it was found that the LP optimal solution was optimal to the QP problem. However, in the CPU time challenges were observed with the BODYD2 for the proposed heuristic and as a result we could not accurately obtain the necessary CPU time for these two cases. There was no challenge with the other two methods on the same BODYD2 problem. This experiment was conducted twice, but the same observation. We have no reason to support this behaviour but we believe it may be due to some local computational environment.

\section{Conclusion}

The convex QP problem can be solved like a linear programming problem efficiently either by the simplex method or the interior point algorithm. The restricted base entry is not necessary by the proposed approach. Complementary slackness can retard the simplex method, which is roughly eight times slower than the full speed simplex method. Taking complementary slackness conditions away itself is a big reduction in the number of constraints in the proposed linear formulation of the quadratic programming problem. More experiments are likely to give more insight and advantages of the proposed approach. The proposed method is in fact the usual simplex method applied to solving an ordinary LP that was obtained from the given convex QP. Also note that a large number of Maros-Maszaros test problems are giving rise to small to medium size LPs and therefore the proposed method dominates solving a large number of QPs, as is reflected in Table 1. From these results, it may be noted that, for example in the case of medium sized problems at serial 118 to 124 and large sized problems at serial number 125 to 132, the proposed heuristic outperformed the other two with respect to the cpu time. 


\section{Acknowledgements}

The authors are thankful to the referees for their helpful and constructive comments.

\section{References}

[1] Gupta, O.K. (1995) Applications of Quadratic Programming. Journal of Information and Optimization Sciences, 16, 177-194. http://dx.doi.org/10.1080/02522667.1995.10699213

[2] Horst, R., Pardalos, P.M. and Thoai, N.V. (2000) Introduction to Global Optimization: Non-Convex Optimization and Its Applications. Kluwer Academic Publishers, Dordrecht. http://dx.doi.org/10.1007/978-1-4615-0015-5

[3] McCarl, B.A., Moskowitz, H. and Furtan, H. (1977) Quadratic Programming Applications. Omega, 5, 43-55. http://dx.doi.org/10.1016/0305-0483(77)90020-2

[4] Burer, S.D. and Vandenbussche, D. (2008) A Finite Branch and Bound Algorithm for Non-Convex Quadratic Programs with Semidefinite Relaxations. Mathematical Programming Series A, 113, 259-282. http://dx.doi.org/10.1007/s10107-006-0080-6

[5] Burer, S.D. and Vandenbussche, D. (2009) Globally Solving Box-Constrained Non Convex Quadratic Programs with Semidefinite-Based Finite Branch-and-Bound. Computational Optimisation and Applications, 43, 181-195.

[6] Freund, R.M. (2002) Solution Methods for Quadratic Optimization. Lecture Notes, Massachusetts Institute of Technology, Cambridge, MA.

[7] Gondzio, J. (2012) Interior Point Methods 25 Years Later. European Journal of Operational Research, 218, 587-601. http://dx.doi.org/10.1016/j.ejor.2011.09.017

[8] More, J.J. and Toraldo, G. (1989) Algorithms for Bound Constrained Quadratic Programming Problems. Numerische Mathematik, 55, 377-400. http://dx.doi.org/10.1007/BF01396045

[9] Liu, S.T. and Wang, R.T. (2007) A Numerical Solution Method to Interval Quadratic Programming. Applied Mathematics and Computations, 189, 1274-1281. http://dx.doi.org/10.1016/j.amc.2006.12.007

[10] Maes, C. and Saunders, M. (2012) A Regularized Active-Set Method for Sparse Convex Quadratic Programming. 21st International Symposium on Mathematical Programming, Berlin, 19-24 August 2012.

[11] Jensen, P.A. and Bard, J.F. (2012) Operations Research Models and Methods. John Wiley \& Sons Inc., Hoboken.

[12] Lee, C.R. (2011) Unit 8: Quadratic Programming Active set Method and Sequential Quadratic Programming. http://www.cs.nthu.edu.tw/ cherung/teaching/2011cs5321/handout8.pdf

[13] Winston, W.L. (2004) Operations Research Applications and Algorithms. 4th Edition, Duxbury Press, Pacific Grove, CA.

[14] Maros, I. and Meszaros, C. (1999) A Repository of Convex Quadratic Programming Problems. Optimization Methods and Software, 11-12, 671-681. 